\title{
SOME REMARKS ON THE DETERMINATION OF QUANTUM STATES BY MEASUREMENTS
}

\author{
Werner Stulpe \\ Max-Planck-Institut für Biophysikalische Chemie \\ D-3400 Göttingen 1, Federal Republic of Germany \\ Matthias Singer \\ Institut für Theoretische Physik \\ Technische Universität Berlin \\ D-1000 Berlin 12, Federal Republic of Germany
}

Received July 21, 1989; revised January 22, 1990

The problem of state determination of quantum systems by the probability distributions of some observables is considered. In particular, we review a question already asked by $W$. Pauli, namely, the determination of pure states of spinless particles by the distributions of position and momentum. In this context we give a new example of two wave functions differing by a piecewise constant phase having the same position and momentum distributions. The Pauli problem is investigated also under incorporation of special types of the Hamiltonian. Moreover, in case of spin-1 systems with three-dimensional Hilbert space, it is shown that the probabilities for the values of six suitably chosen spin components determine their state.

Key words: state determination, Pauli problem, spin-1 systems.

\section{INTRODUCTION}

In his famous articles of 1933 and 1958 in the Encyclopedia of Physics, W. Pauli [1] asked the question whether the wave function of spinless particles is determined up to a constant phase factor by the corresponding position and momentum probability density. He wrote that this problem had not been investigated yet. Meanwhile, the question has been treated in some papers and answered negatively by easy counter-examples [2-7]. However, these results seem to be not very well known. One aim of our paper is to review the work 
done on the Pauli problem and a second one to put it into a more general context.

Consider conventional quantum mechanics without superselection rules, where the density operators $W$ in Hilbert space $\mathbf{H}$ represent the states of quantum systems $\left(W=W^{*}, W \geq 0, \operatorname{tr} W=1\right)$. The observables are usually described by the self-adjoint operators in $\mathbf{H}$ or, equivalently, by the corresponding spectral measures which are projection-valued measures defined on the Borel sets of the real line $\mathbf{R}$. More generally, an observable taking values in $\mathbf{R}^{N}$ is described by a projection-valued measure $B \rightarrow E(B)$, where $B$ is a Borel set of $\mathbf{R}^{N}$ and $E(B)$ an orthogonal projection in $\mathbf{H}$. Such a measure has the properties

(i) $E(\emptyset)=0, E\left(\mathbf{R}^{N}\right)=1$,

(ii) $E\left(\bigcup_{j=1}^{\infty} B_{j}\right)=\sum_{j=1}^{\infty} E\left(B_{j}\right)$ for $B_{j} \cap B_{k}=\emptyset(j \neq k)$,

the infinite sum being understood as strong limit of partial sums. (It turns out that the concept of projection-valued measures is based on a restrictive view of quantum measurement. The sufficiently general concept of positive-operator-valued measures will briefly be discussed in Section 4.) The probability that a measurement of the observable $E$ performed on systems in state $W$ yields values in a Borel set $B$ is

$$
P_{W}^{E}(B):=\operatorname{tr} W E(B) \text {. }
$$

$B \rightarrow P_{W}^{E}(B)$ is a probability measure on the Borel sets of $\mathbf{R}^{N}$, which we call the probability distribution of the observable $E$ in the state $W$.

The extreme points of the convex set of all density operators are the one-dimensional projections, i.e., the pure states correspond to the projections $|\psi><\psi|$ resp. to the unit vectors $\psi \in \mathbf{H}$. From Eq. (1.1), it follows that

$$
P_{\psi}^{E}(B):=P_{|\psi><\psi|}^{E}(B)=\langle\psi| E(B) \psi>.
$$

Remember that two unit vectors differing by a phase factor $e^{i \alpha}$, $\alpha \in \mathbf{R}$, represent the same state.

Let $M$ be a set of observables where we do not suppose that each projection-valued measure is defined on the same $\mathbf{R}^{N}$. For a given state $W, M$ yields a set of probability measures $P_{W}^{E}$. It may happen that any two different states always give rise to different sets of probability distributions. In this case, an arbitrary state $W$ is uniquely determined by the distributions $P_{W}^{E}$ of all observables $E \in M$, i.e. $P_{W_{1}}^{E}(B)=P_{W_{2}}^{E}(B)$ for every $E \in M$ and for all Borel 
sets $B$ of the corresponding $\mathbf{R}^{N}$ implies the equality of the two states $W_{1}$ and $W_{2}$.

For example, let $M$ be the set of the spectral measures of all one-dimensional projection operators $P_{\phi}:=|\phi\rangle\langle\phi|, \phi \in \mathbf{H}$, $\|\phi\|=1$, and assume that all these observables have the same probability distributions in the two states $W_{1}$ and $W_{2}$. The spectral measure of $P_{\phi}$ has just the value $P_{\phi}$ for any Borel set of $\mathbf{R}$ that contains the number 1 , but not 0 . According to (1.1), we obtain for such Borel sets

$$
\operatorname{tr} W_{1} P_{\phi}=\operatorname{tr} W_{2} P_{\phi} \text {. }
$$

Hence, $\left\langle\phi \mid W_{1} \phi\right\rangle=\left\langle\phi \mid W_{2} \phi\right\rangle$ holds for all unit vectors $\phi \in \mathbf{H}$, and the probability distributions of all observables of $M$ in any state determine this state uniquely.

Now let $M$ consist only of the one-dimensional projection operators $P_{\phi_{\nu}}$ corresponding to a complete orthonormal system $\left\{\phi_{\nu}\right\}_{\nu \in \mathrm{N}}$. This example fails for state determination even if one considers only pure states. In fact, by insertion of $W:=P_{\psi}=|\psi\rangle\langle\psi|$ into $\operatorname{tr} W P_{\phi}$ and replacement of $P_{\phi}$ by $P_{\phi_{\nu}}$ we see that only the moduli of the coefficients $a_{\nu}$ in $\psi=\sum_{\nu=1}^{\infty} a_{\nu} \phi_{\nu}$ are determined by the probability distributions of the observables of $M$. The set $M=\left\{P_{\phi_{\nu}} \mid \nu \in \mathrm{N}\right\}$ is equivalent to a set which consists of a single observable given by a self-adjoint operator $A$ with spectral representation $A=\sum_{\nu=1}^{\infty} \lambda_{\nu} P_{\phi_{\nu}}$ $\left(\lambda_{\nu} \neq \lambda_{\mu}\right.$ for $\left.\nu \neq \mu\right)$.

From the physical point of view, state determination as described is interesting only if the set $M$ consists of only finitely many observables. For reasons of simplicity, we restrict our investigations to pure states, so that the problems treated in this paper are of the following type. Given a finite set $\left\{E_{1}, E_{2}, \ldots, E_{n}\right\}$ of observables, do the probability distributions $B \rightarrow P_{\psi}^{E_{j}}(B)$ determine the pure state uniquely? More precisely, if $P_{\psi_{1}}^{E_{j}}(B)=P_{\psi_{2}}^{E_{j}}(B)$ is fulfilled for every $j=1,2, \ldots, n$ and for all Borel sets $\mathrm{B}$ of the corresponding $\mathbf{R}^{N}$, where $\psi_{1}, \psi_{2} \in \mathbf{H}$ are unit vectors, does then $\psi_{2}=e^{i \alpha} \psi_{1}, \alpha \in \mathbf{R}$, hold true?

Let us discuss the physical meaning of our investigations. Systems in an arbitrary state $W$ represent a statistical ensemble and are the result of a preparation procedure performed on the systems. In particular, it is possible to produce arbitrarily (but finitely!) many systems of a given state. The measurement of an observable $E$ on many systems of an ensemble $W$ yields the probability distribution $P_{W}^{E}$ in physical approximation. Hence, if the finitely many observables $E_{1}, E_{2}, \ldots, E_{n}$ determine pure states, then an unknown pure state can approximately be determined experimentally, namely, by the measurement of the distributions $P_{\psi}^{E_{j}}$.

We emphasize that we consider the determination of an arbitrary given state by measurements and not the preparation of a certain 
state by measurements. Roughly speaking, the latter preparative measurements (as described in an idealized manner by von Neumann's projection postulate) concern states after measurement whereas our considerations concern states before measurement.

In Section 2, we review the work done on the Pauli problem and add a new counter-example involving two wave functions differing by a piecewise constant phase. It is shown that for free particles, harmonic oscillators, and particles moving in an attractive Coulomb potential, even the probability distributions of position, momentum, and energy are not sufficient to determine pure states uniquely. In Section 3 we review the already known result that states of spin- $\frac{1}{2}$ systems with two-dimensional Hilbert space are determined by the probabilities for the measuring values of three orthogonal spin components. Moreover, we investigate spin-1 systems with three-dimensional Hilbert space and show that their pure states can be determined by six suitably chosen spin components. Finally, in Section 4 we give some remarks concerning observables represented by positive-operator-valued measures.

\section{COUNTER-EXAMPLES FOR STATE DETER- MINATION BY POSITION, MOMENTUM, AND HAMILTONIAN}

Let us consider spinless particles with the space $\mathbf{L}^{2}\left(\mathbf{R}^{3}\right)$ of squareintegrable functions as its Hilbert space $\mathrm{H}$. The three-dimensional position observable is described by the projection-valued measure $E^{Q}$ defined on the Borel sets $B$ of $\mathrm{R}^{3}$ by

$$
E^{Q}(B) \psi:=\chi_{B} \psi,
$$

where $\psi \in \mathbf{L}^{2}\left(\mathbf{R}^{3}\right)$ and $\chi_{B}(x):=1$ if $x \in B$ and $\chi_{B}(x):=0$ otherwise. The three-dimensional momentum observable $E^{P}$ is given by

$$
E^{P}(B):=F^{-1} E^{Q}(B) F,
$$

where $F$ is the unitary operator of Fourier transformation in $L^{2}\left(\mathbf{R}^{3}\right)$. According to Eq. (1.2), we obtain

$$
P_{\psi}^{E^{Q}}(B)=<\left.\psi\left|E^{Q}(B) \psi>=\int_{B}\right| \psi(x)\right|^{2} d^{3} x
$$

and

$$
P_{\psi}^{E^{P}}(B)=\left\langle\psi\left|E^{P}(B) \psi>=<F \psi\right| E^{Q}(B) F \psi>=\int_{B}|\tilde{\psi}(\bar{k})|^{2} d^{3} k\right.
$$


for the position and momentum probabilities, $\tilde{\psi}:=F \psi$. The observables $E^{Q}$ and $E^{P}$ do not correspond to self-adjoint operators, but their marginal observables do. For instance, $b \rightarrow E^{Q_{1}}(b):=E^{Q}(b \times \mathbf{R} \times \mathbf{R})$ where $b$ is a Borel set of $\mathbf{R}$ is just the spectral measure of the operator $Q_{1}$ of the first position coordinate, $\left(Q_{1} \psi\right)(x):=x_{1} \psi(x)$.

Now we present some counter-examples which show that a pure state $\psi \in \mathbf{L}^{2}\left(\mathbf{R}^{3}\right),\|\psi\|=1$, is in general not uniquely determined by the probability distributions of position and momentum resp. by its position probability density $|\psi|^{2}$ and momentum probability density $|\tilde{\psi}|^{2}$. To that end, let $\psi$ be a wave function fulfilling the conditions

(i) $\operatorname{Re} \psi$ and $\operatorname{Im} \psi$ are linearly independent,

(ii) $\psi$ is either even, i.e. $\psi(x)=\psi(-x)$ for all $x \in \mathbf{R}^{3}$, or odd, i.e. $\psi(x)=-\psi(-x)$.

Define $\psi_{1}:=\psi$ and $\psi_{2}:=e^{i \beta} \bar{\psi}$ where $\beta \in \mathbf{R}$ and the bar denotes complex conjugation. Then, according to (i), the wave functions $\psi_{1}$ and $\psi_{2}$ are inequivalent, i.e. $\psi_{2} \neq e^{i \alpha} \psi_{1}$ for every $\alpha \in \mathbf{R}$. From their definition it follows that $\left|\psi_{1}\right|^{2}=\left|\psi_{2}\right|^{2}$ and $\tilde{\psi}_{2}(k)=e^{i \beta} \overline{\tilde{\psi}_{1}(-k)}$ for all $k \in \mathbf{R}^{3}$. By condition (ii), the latter equality implies $\tilde{\psi}_{2}(k)$ $= \pm e^{i \beta} \tilde{\psi}_{1}(k)$ and $\left|\tilde{\psi}_{1}\right|^{2}=\left|\tilde{\psi}_{2}\right|^{2}$. Hence, we have constructed a whole class of counter-examples.

The same counter-example was given by V. Bargmann some 40 years ago and published in the book of $H$. Reichenbach [2]. As far as the authors of this paper know, it was the first counter-example concerning state determination by position and momentum that appeared the literature. In 1968, W. Gale et al. [3] gave a counterexample which is a special case of our general one, namely

$$
\begin{aligned}
& \psi_{1}(x):=R(r) Y_{l m}(\theta, \phi), \\
& \psi_{2}(x):=R(r) Y_{l,-m}(\theta, \phi),
\end{aligned}
$$

where $r, \theta, \phi$ are the polar coordinates of the point $x, Y_{l m}$ the spherical harmonics, $R$ is any real-valued suitably normalized radial function, and $m \neq 0$. In fact, because of $Y_{l m}(\theta, \phi)=(-1)^{l} Y_{l m}(\pi-\theta, \phi+\pi)$, $\psi_{1}$ is an even or odd function, and $\psi_{2}$ coincides with $\overline{\psi_{1}}$ up to a phase factor.

In 1977, E. Prugovečki [4] presented a counter-example which is slightly more general than ours, similar considerations also involving the Hamiltonian were done by A. Vogt [5] in the same year. In 1978 , J. V. Corbett and C. A. Hurst [6] presented a mathematically systematic paper on some aspects of the Pauli problem. The next 
example is a special case of ours and was published by B. Z. Moroz in 1983 [7]. Let

$$
\begin{aligned}
& \psi_{1}(x):=f(r) e^{i g(r)}, \\
& \psi_{2}(x):=f(r) e^{-i g(r)},
\end{aligned}
$$

where $f$ and $g$ are real-valued functions, the latter one being not constant, and $r=\|x\|=\sqrt{x_{1}^{2}+x_{2}^{2}+x_{3}^{2}}$. This example shows that even the distributions of position, momentum, and the three components of angular momentum do not determine pure states. Further interesting information can be found in an article of $\mathrm{H}$.-W. Wiesbrock from 1987 [8].

The following counter-example, due to the authors, consists of two wave functions differing by a piecewise constant phase function. Namely, let $\phi \in \mathrm{L}^{2}(\mathbf{R})$ be a function such that $\int|\phi(x)|^{2} d x=\frac{1}{3}$ and $\phi(x)=0$ for $x<0$ and $x \geq a, a>0$. Define two wave functions on one-dimensional configuration space by

$$
\begin{aligned}
& \psi_{1}(x):=\phi(x+a)+\phi(x)-\phi(x-a), \\
& \psi_{2}(x):=\phi(x+a)-\phi(x)-\phi(x-a) .
\end{aligned}
$$

Then the corresponding position probability densities are equal, the Fourier transforms are given by

$$
\begin{aligned}
& \tilde{\psi}_{1}(k)=\left(e^{i k a}+1-e^{-i k a}\right) \tilde{\phi}(k), \\
& \tilde{\psi}_{2}(k)=\left(e^{i k a}-1-e^{-i k a}\right) \tilde{\phi}(k),
\end{aligned}
$$

and the momentum probability densities also coincide:

$$
\left|\tilde{\psi}_{1}(k)\right|^{2}=\left|\tilde{\psi}_{2}(k)\right|^{2}=\left(1+4 \sin ^{2} k a\right)|\tilde{\phi}(k)|^{2} .
$$

Note that $\psi_{1}$ and $\psi_{2}$ may be real-valued wave functions.

Next we investigate the problem of state determination by the probability distributions of position, momentum, and energy. For free particles of mass $m$, the Hamiltonian can be defined as

$$
H:=F^{-1} \tilde{H} F,
$$

where the multiplication operator $\tilde{H}$ is given by

$$
(\tilde{H} \tilde{\psi})(k):=\frac{\|k\|^{2}}{2 m} \tilde{\psi}(k) \text {. }
$$

Here $\|k\|$ denotes the Euclidean norm of $k \in \mathbf{R}^{3}$, and $\tilde{\psi} \in \mathbf{L}^{2}\left(\mathbf{R}^{3}\right)$ is any element of the domain of $\tilde{H}$; the domains of the self-adjoint 
operators $H$ and $\tilde{H}$ are obvious. From (2.5) and (2.6) it follows that the spectral measure $E^{H}$ of $H$ is given by

$$
E^{H}(b)=F^{-1} E^{Q}\left(h^{-1}(b)\right) F=E^{P}\left(h^{-1}(b)\right),
$$

where $b$ is a Borel set of $\mathbf{R}, E^{Q}$ and $E^{P}$ are the projection-valued measures according to (2.1) and (2.2), $h$ is the function $k \rightarrow h(k)$ $:=\|k\|^{2} / 2 m$ from $\mathbf{R}^{3}$ into $\mathbf{R}$, and $h^{-1}(b):=\left\{k \in \mathbf{R}^{3} \mid h(k) \in b\right\}$. Equations (1.2) and (2.7) yield

$$
P_{\psi}^{E^{H}}(b)=<\left.\psi\left|E^{H}(b) \psi>=\int_{h^{-1}(b)}\right| \tilde{\psi}(k)\right|^{2} d^{3} k
$$

for the probability that the energy of the free particles in state $\psi$ has a value in the set $b, \tilde{\psi}=F \psi$. Hence, if two states give rise to the same momentum probability density, they also give the same energy probability distribution $P_{\psi}^{E^{H}}$. Thus, the states of free particles are not determined by position, momentum, and energy.

In the case of one-dimensional or (possibly inisotropic) threedimensional harmonic oscillators, choose wave functions $\psi_{1}=\psi$ and $\psi_{2}=e^{i \beta} \bar{\psi}$ with $\psi$ satisfying the conditions (i) and (ii). Since the eigenfunctions of harmonic oscillators are essentially real-valued, the probability distributions of $E^{Q}, E^{P}$, and $E^{H}$ coincide in both states. If an observable is represented by a self-adjoint operator having a complete orthonormal system of real-valued eigenfunctions, then position, momentum, and this observable do generally not determine pure states.

Finally, look at the eigenfunctions $\psi_{n l m}$ and $\psi_{n l,-m}$ of hydrogen atom electrons. These eigenfunctions are of the form (2.3) and (2.4). For $m \neq 0$, they yield the same position and momentum probability density and the same energy, but they differ with respect to the angular momentum.

\section{STATE DETERMINATION OF SPIN SYSTEMS}

In this section we discuss the state determination of spin- $\frac{1}{2}$ and spin-1 systems where the Hilbert space is two- resp. three-dimensional. We first show that the states of spin $-\frac{1}{2}$ systems are determined by the probability distributions of three mutually orthogonal spin components.

Consider the operators of the three spin components with respect to the coordinate axes and denote the normalized eigenvectors corresponding to the eigenvalues $m= \pm \frac{1}{2}$ by $u_{m}, v_{m}$, and $\phi_{m}$, respectively. 
These eigenvectors are unique up to phase factors which are usually be chosen such that

$$
\begin{aligned}
& u_{\frac{1}{2}}=\frac{1}{\sqrt{2}}\left(\phi_{\frac{1}{2}}+\phi_{-\frac{1}{2}}\right), \quad u_{-\frac{1}{2}}=\frac{1}{\sqrt{2}}\left(\phi_{\frac{1}{2}}-\phi_{-\frac{1}{2}}\right), \\
& v_{\frac{1}{2}}=\frac{1}{\sqrt{2}}\left(\phi_{\frac{1}{2}}+i \phi_{-\frac{1}{2}}\right), \quad v_{-\frac{1}{2}}=\frac{1}{\sqrt{2}}\left(\phi_{\frac{1}{2}}-i \phi_{-\frac{1}{2}}\right) .
\end{aligned}
$$

Suppose now that the probabilities for the measuring values $m$ of the spin components coincide in two states $\psi_{1}$ and $\psi_{2}$ :

$$
\begin{aligned}
& \left|<u_{m}\right| \psi_{1}>\left.\right|^{2}=\left|<u_{m}\right| \psi_{2}>\left.\right|^{2}, \\
& \left|<v_{m}\right| \psi_{1}>\left.\right|^{2}=\left|<v_{m}\right| \psi_{2}>\left.\right|^{2}, \\
& \left|<\phi_{m}\right| \psi_{1}>\left.\right|^{2}=\left|<\phi_{m}\right| \psi_{2}>\left.\right|^{2}
\end{aligned}
$$

( $m= \pm \frac{1}{2}$ ). Equation (3.5) implies

$$
<\phi_{m}\left|\psi_{2}>=e^{i \alpha_{m}}<\phi_{m}\right| \psi_{1}>,
$$

where $\alpha_{m} \in \mathbf{R}$. Setting $u_{m}=u_{\frac{1}{2}}$ in (3.3), inserting the expression (3.1) for $u_{\frac{1}{2}}$, and taking account of (3.6), it follows that

$$
\begin{gathered}
\frac{1}{2}\left|<\phi_{\frac{1}{2}}\right| \psi_{1}>\left.\right|^{2}+\frac{1}{2}\left|<\phi_{-\frac{1}{2}}\right| \psi_{1}>\left.\right|^{2}+\operatorname{Re}\left(<\phi_{\frac{1}{2}}\left|\psi_{1}><\psi_{1}\right| \phi_{-\frac{1}{2}}>\right) \\
=\frac{1}{2}\left|<\phi_{\frac{1}{2}}\right| \psi_{1}>\left.\right|^{2}+\frac{1}{2}\left|<\phi_{-\frac{1}{2}}\right| \psi_{1}>\left.\right|^{2} \\
+\operatorname{Re}\left(\exp \left(i\left(\alpha_{\frac{1}{2}}-\alpha_{-\frac{1}{2}}\right)\right)<\phi_{\frac{1}{2}}\left|\psi_{1}><\psi_{1}\right| \phi_{-\frac{1}{2}}>\right) .
\end{gathered}
$$

If we assume $<\phi_{\frac{1}{2}}\left|\psi_{1}><\psi_{1}\right| \phi_{-\frac{1}{2}}>\neq 0$ and write this term as $r e^{i \beta}$ $(r, \beta \in \mathbf{R}, r>0)$, then the last equality yields

$$
\cos \left(\beta+\alpha_{\frac{1}{2}}-\alpha_{-\frac{1}{2}}\right)=\cos \beta .
$$

Modulo $2 \pi$, this equation has two solutions for $\alpha_{\frac{1}{2}}-\alpha_{-\frac{1}{2}}$ unless $\beta=0$. Now take Eq. (3.4) into account. By an analogous procedure involving (3.2) and (3.6), we obtain

$$
\sin \left(\beta+\alpha_{\frac{1}{2}}-\alpha_{-\frac{1}{2}}\right)=\sin \beta .
$$

Hence, by (3.7) and (3.8), $\alpha_{\frac{1}{2}}=\alpha_{-\frac{1}{2}}+2 \pi k, k \in Z$. Thus, the unit vectors $\psi_{1}$ and $\psi_{2}$ differ only by a phase factor. This result is also true if $<\phi_{\frac{1}{2}}\left|\psi_{1}><\psi_{1}\right| \phi_{-\frac{1}{2}}>=0$. 
The result just obtained can also be proved by the fact that a density operator $W$ in two-dimensional Hilbert space has a representation $W=\frac{1}{2}(1+w \cdot \sigma)$ where 1 is the unit operator, $w \in \mathbf{R}^{3}$ with Euclidean Norm $\|w\| \leq 1$, and $w \cdot \sigma:=\sum_{l=1}^{3} w_{l} \sigma_{l}$. Moreover, this shows that three orthogonal spin $-\frac{1}{2}$ components determine even mixed states (cf. $[19,21])$.

To determine the states of spin-1 systems, the consideration of more than three spin components is necessary. Let $e_{l}, l=1,2,3$, be the unit vectors with respect to the coordinate axes, $n$ any unit vector in $\mathbf{R}^{3}$, and $S_{l}$ the $e_{l}$-component of 1 -spin. The observable of spin $n$-component is represented by the operator $n \cdot S:=\sum_{l=1}^{3} n_{l} S_{l}$ where $n=\sum_{l=1}^{3} n_{l} e_{l}$. Denoting the normalized eigenvectors of $n \cdot S$ corresponding to the eigenvalues $m=1,0,-1$ by $u_{m}^{n}$ and especially those of $S_{3}$ by $\phi_{m}$, the former ones can be written as

$$
\begin{aligned}
& u_{1}^{n}=\frac{1}{2} \sqrt{1-n_{3}^{2}}\left(\frac{n_{1}+i n_{2}}{1-n_{3}} \phi_{1}+\sqrt{2} \phi_{0}+\frac{n_{1}-i n_{2}}{1+n_{3}} \phi_{-1}\right), \\
& \text { if } n_{3} \neq \pm 1 \text {, } \\
& u_{0}^{n}=\frac{1}{\sqrt{2}}\left(\frac{\left|n_{3}\right|}{n_{3}}\left(n_{1}+i n_{2}\right) \phi_{1}-\sqrt{2}\left|n_{3}\right| \phi_{0}\right. \\
& \left.-\frac{\left|n_{3}\right|}{n_{3}}\left(n_{1}-i n_{2}\right) \phi_{-1}\right), \quad \text { for } n_{3} \neq 0, \\
& u_{0}^{n}=\frac{1}{\sqrt{2}}\left(\left(n_{1}+i n_{2}\right) \phi_{1}-\left(n_{1}-i n_{2}\right) \phi_{-1}\right), \text { for } n_{3}=0 \text {, } \\
& u_{-1}^{n}=\frac{1}{2} \sqrt{1-n_{3}^{2}}\left(-\frac{n_{1}+i n_{2}}{1+n_{3}} \phi_{1}+\sqrt{2} \phi_{0}-\frac{n_{1}-i n_{2}}{1-n_{3}} \phi_{-1}\right), \\
& \text { if } n_{3} \neq \pm 1 \text {. }
\end{aligned}
$$

We show now that the probability distributions of six suitably chosen spin components determine a pure state of spin-1 systems. Suppose two states $\psi_{1}$ and $\psi_{2}$ satisfy

$$
\left|<u_{m}^{n}\right| \psi_{1}>\left.\right|^{2}=\left|<u_{m}^{n}\right| \psi_{2}>\left.\right|^{2},
$$

for $m=1,0,-1$, and $n=e_{1}, e_{2}, e_{3}, \frac{1}{\sqrt{2}}\left(e_{1}+e_{2}\right), \frac{1}{\sqrt{2}}\left(e_{1}+e_{3}\right), \frac{1}{\sqrt{2}}\left(e_{2}+e_{3}\right)$. For $n=e_{3}$, Eq. (3.10) implies

$$
<\phi_{m} \mid \psi_{2}>=e^{i \alpha_{m}} a_{m}
$$

where $\alpha_{m} \in \mathbf{R}$ and $a_{m}:=\left\langle\phi_{m} \mid \psi_{1}\right\rangle$. If $n$ is equal to one of the other five vectors, we insert the respective expression for $u_{m}^{n}$ given by Eqs. (3.9) into (3.10) and take account of (3.11). After some short 
calculations, we obtain

$$
\begin{aligned}
& \operatorname{Re}\left(\sqrt{2} a_{1} \overline{a_{0}}+\sqrt{2} a_{0} \overline{a_{-1}}+a_{1} \overline{a_{-1}}\right) \\
& \quad=\operatorname{Re}\left(\sqrt{2} e^{i\left(\alpha_{1}-\alpha_{0}\right)} a_{1} \overline{a_{0}}+\sqrt{2} e^{i\left(\alpha_{0}-\alpha_{-1}\right)} a_{0} \overline{a_{-1}}+e^{i\left(\alpha_{1}-\alpha_{-1}\right)} a_{1} \overline{a_{-1}}\right), \\
& \text { for } n=e_{1}, m=1 ;
\end{aligned}
$$

$\operatorname{Re} a_{1} \overline{a_{-1}}=\operatorname{Re}\left(e^{i\left(\alpha_{1}-\alpha_{-1}\right)} a_{1} \overline{a_{-1}}\right)$, for $n=e_{1}, m=0$;

$$
\begin{aligned}
& \operatorname{Re}\left(i \sqrt{2} a_{1} \overline{a_{0}}+i \sqrt{2} a_{0} \overline{a_{-1}}+a_{1} \overline{a_{-1}}\right) \\
& \quad=\operatorname{Re}\left(i \sqrt{2} e^{i\left(\alpha_{1}-\alpha_{0}\right)} a_{1} \overline{a_{0}}+i \sqrt{2} e^{i\left(\alpha_{0}-\alpha_{-1}\right)} a_{0} \overline{a_{-1}}+e^{i\left(\alpha_{1}-\alpha_{-1}\right)} a_{1} \overline{a_{-1}}\right),
\end{aligned}
$$

for $n=e_{2}, m=1$;

$\operatorname{Re} a_{1} \overline{a_{-1}}=\operatorname{Re}\left(e^{i\left(\alpha_{1}-\alpha_{-1}\right)} a_{1} \overline{a_{-1}}\right)$, for $n=e_{2}, m=0$;

$$
\begin{aligned}
& \operatorname{Re}\left((1-i) a_{1} \overline{a_{0}}+(1-i) a_{0} \overline{a_{-1}}-i a_{1} \overline{a_{-1}}\right) \\
& =\operatorname{Re}\left((1-i) e^{i\left(\alpha_{1}-\alpha_{0}\right)} a_{1} \overline{a_{0}}+(1-i) e^{i\left(\alpha_{0}-\alpha_{-1}\right)} a_{0} \overline{a_{-1}}\right. \\
& \left.\quad-i e^{i\left(\alpha_{1}-\alpha_{-1}\right)} a_{1} \overline{a_{-1}}\right), \text { for } n=\frac{1}{\sqrt{2}}\left(e_{1}+e_{2}\right), m=1
\end{aligned}
$$

$\operatorname{Re} i a_{1} \overline{a_{-1}}=\operatorname{Re}\left(i e^{i\left(\alpha_{1}-\alpha_{-1}\right)} a_{1} \overline{a_{-1}}\right)$, for $n=\frac{1}{\sqrt{2}}\left(e_{1}+e_{2}\right), m=0$;

$$
\begin{aligned}
& \operatorname{Re}\left((2+\sqrt{2}) a_{1} \overline{a_{0}}+(2-\sqrt{2}) a_{0} \overline{a_{-1}}+a_{1} \overline{a_{-1}}\right) \\
& =\operatorname{Re}\left((2+\sqrt{2}) e^{i\left(\alpha_{1}-\alpha_{0}\right)} a_{1} \overline{a_{0}}+(2-\sqrt{2}) e^{i\left(\alpha_{0}-\alpha_{-1}\right)} a_{0} \overline{a_{-1}}\right. \\
& \left.\quad+e^{i\left(\alpha_{1}-\alpha_{-1}\right)} a_{1} \overline{a_{-1}}\right), \text { for } n=\frac{1}{\sqrt{2}}\left(e_{1}+e_{3}\right), m=1 ;
\end{aligned}
$$

$$
\begin{aligned}
& \operatorname{Re}\left(\sqrt{2} a_{1} \overline{a_{0}}-\sqrt{2} a_{0} \overline{a_{-1}}+a_{1} \overline{a_{-1}}\right) \\
& =\operatorname{Re}\left(\sqrt{2} e^{i\left(\alpha_{1}-\alpha_{0}\right)} a_{1} \overline{a_{0}}-\sqrt{2} e^{i\left(\alpha_{0}-\alpha_{-1}\right)} a_{0} \overline{a_{-1}}+e^{i\left(\alpha_{1}-\alpha_{-1}\right)} a_{1} \overline{a_{-1}}\right) \\
& \text { for } \quad n=\frac{1}{\sqrt{2}}\left(e_{1}+e_{3}\right), m=0 ; \\
& \operatorname{Re}\left(i(2+\sqrt{2}) a_{1} \overline{a_{0}}+i(2-\sqrt{2}) a_{0} \overline{a_{-1}}+a_{1} \overline{a_{-1}}\right) \\
& \quad=\operatorname{Re}\left(i(2+\sqrt{2}) e^{i\left(\alpha_{1}-\alpha_{0}\right)} a_{1} \overline{a_{0}}+i(2-\sqrt{2}) e^{i\left(\alpha_{0}-\alpha_{-1}\right)} a_{0} \overline{a_{-1}}\right. \\
& \left.\quad+e^{i\left(\alpha_{1}-\alpha_{-1}\right)} a_{1} \overline{a_{-1}}\right), \text { for } n=\frac{1}{\sqrt{2}}\left(e_{2}+e_{3}\right), m=1 ;
\end{aligned}
$$




$$
\begin{aligned}
& \operatorname{Re}\left(i \sqrt{2} a_{1} \overline{a_{0}}-i \sqrt{2} a_{0} \overline{a_{-1}}+a_{1} \overline{a_{-1}}\right) \\
& \quad=\operatorname{Re}\left(i \sqrt{2} e^{i\left(\alpha_{1}-\alpha_{0}\right)} a_{1} \overline{a_{0}}-i \sqrt{2} e^{i\left(\alpha_{0}-\alpha_{-1}\right)} a_{0} \overline{a_{-1}}+e^{i\left(\alpha_{1}-\alpha_{-1}\right)} a_{1} \overline{a_{-1}}\right), \\
& \text { for } n=\frac{1}{\sqrt{2}}\left(e_{2}+e_{3}\right), m=0 .
\end{aligned}
$$

The corresponding equations for $m=-1$ give no further information.

From (3.12), (3.13), and (3.14) it follows that

$$
a_{1} \overline{a_{0}}+a_{0} \overline{a_{-1}}=e^{i\left(\alpha_{1}-\alpha_{0}\right)} a_{1} \overline{a_{0}}+e^{i\left(\alpha_{0}-\alpha_{-1}\right)} a_{0} \overline{\alpha_{-1}}
$$

holds. Equations (3.13) and (3.17) imply

$$
a_{1} \overline{a_{-1}}=e^{i\left(\alpha_{1}-\alpha_{-1}\right)} a_{1} \overline{\alpha_{-1}} .
$$

Equations (3.15) and (3.16) also give no further information. From (3.13), (3.19), and (3.21) we obtain

$$
a_{1} \overline{a_{0}}-a_{0} \overline{a_{-1}}=e^{i\left(\alpha_{1}-\alpha_{0}\right)} a_{1} \overline{a_{0}}-e^{i\left(\alpha_{0}-\alpha_{-1}\right)} a_{0} \overline{\alpha_{-1}}
$$

which also follows from (3.18) and (3.20) by means of (3.12), (3.13), and (3.14). We now have to distinguish three cases:

Case 1: Two of the components $a_{m}$ of $\psi_{1}$ are equal to zero. Then, according to Eq. (3.11), $\psi_{1}$ and $\psi_{2}$ differ only by a phase factor.

Case 2: Exactly one $a_{m}$ is zero. If $a_{1}=0$, then (3.22) implies $\alpha_{0}=\alpha_{-1}$ modulo $2 \pi$, and the states $\psi_{1}$ and $\psi_{2}$ are equivalent again. This result is analogously obtained for the cases $a_{0}=0$ and $a_{-1}=0$, by $(3.23)$ and (3.22), respectively.

Case 3: All three components $a_{m}$ are not zero. Then Eqs. (3.22) and (3.24) yield $\alpha_{1}=\alpha_{0}$ and $\alpha_{0}=\alpha_{-1}$ modulo $2 \pi$. Hence, $\psi_{2}=e^{i \alpha} \psi_{1}$ where $\alpha:=\alpha_{1}$.

Thus, we have proved that a pure state of spin-1 systems is determined by the probabilities for six particular spin components.

Finally, we observe that the probability distributions of even less than six spin components are sufficient for the determination of pure states of spin-1 systems. In Case 3, Eq. (3.23) was not used to obtain the result $\psi_{2}=e^{i \alpha} \psi_{1}$. Hence, (3.17) is unnecessary in this case, and a state is determined by the five spin components corresponding to $n=e_{1}, e_{2}, e_{3}, \frac{1}{\sqrt{2}}\left(e_{1}+e_{3}\right), \frac{1}{\sqrt{2}}\left(e_{2}+e_{3}\right)$. Moreover, it can easily be shown that under the additional assumption $a_{1} \neq a_{-1}$ the result can be concluded from (3.22) and (3.23), i.e. the four spin components with respect to $n=e_{1}, e_{2}, e_{3}, \frac{1}{\sqrt{2}}\left(e_{1}+e_{2}\right)$ are sufficient to determine the state. In Case 2, we have to choose only three suitable components, and in Case 1 solely the observable $S_{3}$ determines the state. In an 
experimental procedure for state determination of spin-1 systems, one should measure the probability distribution of $S_{3}$ first. The result shows which case is present and induces a suitable choice of the other spin components to be measured. The actual state then can in principle be concluded from the measured probability distributions of all these observables.

\section{CONCLUDING REMARKS}

We have shown that in general states of spinless particles are not determined by the distributions of some conventional observables, whereas the distributions of suitable spin components determine states of spin systems. Of course, in this context there are other interesting questions we have not answered. Nevertheless, we finally look at another aspect of state determination.

As already mentioned in the introduction, there are strong physical reasons to replace the definition of a quantum mechanical observable as a projection-valued measure $B \rightarrow E(B)$ as given in Section 1 by a more general one. Namely, an observable taking values in $\mathbf{R}^{N}$ can be described by a positive-operator-valued measure $B \rightarrow F(B)$ having the properties (i) and (ii) of Section 1 where $B$ is a Borel set of $\mathbf{R}^{N}$ and $F(B)$ a positive self-adjoint operator satisfying $0 \leq F(B) \leq 1$ (cf., e.g., $[9,10]$ ). In particular, in this context it is possible to define observables that describe the approximate joint measurement of complementary observables such as position and momentum [11-17] or two orthogonal spin- $\frac{1}{2}$ components [18-21]. Moreover, S. T. Ali, E. Prugovečki, F. E. Schroeck, Jr., and P. Busch $[11,12,18-21]$ discussed observables describing such approximate joint measurements such that the probability distribution of only one observable determines even mixed states uniquely.

\section{ACKNOWLEDGMENTS}

One of us, W. S., wishes to thank Prof. J.-P. Marchand and Prof. S. P. Gudder for interesting and stimulating discussions during his stay at the Mathematics Department of the University of Denver, Denver, Colorado. Moreover, he gratefully acknowledges spiritual support by Prof. Ernst Ruch and a research grant by the DFG, Bonn. 


\section{REFERENCES}

[1] W. Pauli, "Die allgemeinen Prinzipien der Wellenmechanik," in Encyclopedia of Physics, Vol. V, Part I (Springer-Verlag, Berlin, 1958), p. 17; English translation: General Principles of Quantum Mechanics (Springer-Verlag, Berlin, 1980), p. 17.

[2] H. Reichenbach, Philosophic Foundations of Quantum Mechanics (University of California Press, Berkeley, 1948), p. 92.

[3] W. Gale, E. Guth, G. T. Trammell, "Determination of the Quantum State by Measurements," Phys. Rev. 165, 1434-1436 (1968).

[4] E. Prugovečki, "Information-Theoretical Aspects of Quantum Measurement," Int. J. Theor. Phys. 16, 321-331 (1977).

[5] A. Vogt, "Position and Momentum Distributions Do Not Determine the Quantum Mechanical State," in Mathematical Foundations of Quantum Theory, A. R. Marlow, ed. (Academic Press, New York, 1978), pp. 365-372.

[6] J. V. Corbett and C. A. Hurst, "Are Wave Functions Uniquely Determined by Their Position and Momentum Distributions?" J. Austral. Math. Soc. 20 B, 182-201 (1978).

[7] B. Z. Moroz, "Reflections on Quantum Logic," Int. J. Theor. Phys. 22, 329-339 (1983); Erratum, Int. J. Theor. Phys. 23, 497-498 (1984).

[8] H.-W. Wiesbrock, "Born's Postulate and Reconstruction of the $\psi$-Function in Nonrelativistic Quantum Mechanics," Int. J. Theor. Phys. 26, 1175-1184 (1987).

[9] E. B. Davies, Quantum Theory of Open Systems (Academic Press, London, 1976).

[10] G. Ludwig, Foundations of Quantum Mechanics I,II (SpringerVerlag, New York, 1983 and 1985).

[11] S. T. Ali and E. Prugovečki, "Systems of Imprimitivity and Representations of Quantum Mechanics on Fuzzy Phase Spaces," J. Math. Phys. 18, 219-228 (1977).

[12] S. T. Ali and E. Prugovečki, "Classical and Quantum Statistical Mechanics in a Common Liouville Space," Physica 89 A, 501-521 (1977).

[13] F. E. Schroeck, Jr., "A Model of a Quantum Mechanical Treatment of Measurement with a Physical Interpretation," J. Math. Phys. 22, 2562-2572 (1981).

[14] P. Busch and P. J. Lahti, "On Various Joint Measurements of Position and Momentum Observables in Quantum Theory," Phys. Rev. D 29, 1634-1646 (1984).

[15] P. Busch, "Indeterminacy Relations and Simultaneous Measurements in Quantum Theory," Int. J. Theor. Phys. 24, 63-92 (1985). 
[16] E. Prugovečki, Stochastic Quantum Mechanics and Spacetime (Reidel, Dordrecht, 1984).

[17] C. Y. She and H. Heffner, "Simultaneous Measurement of Noncommuting Observables," Phys. Rev. 152, 1103-1110 (1966).

[18] E. Prugovečki, "On Fuzzy Spin Spaces," J. Phys. A: Math. Gen. 10, 543-549 (1977).

[19] F. E. Schroeck, Jr., "On the Stochastic Measurement of Incompatible Spin Components," Found. Phys. 12, 479-497 (1982).

[20] P. Busch, "Unsharp Reality and Joint Measurements for Spin Observables," Phys. Rev. D 33, 2253-2261 (1986).

[21] P. Busch, "Some Realizable Joint Measurements of Complementary Observables," Found. Phys. 17, 905-937 (1987). 